\title{
Detecting the progenitors of core-collapse supernovae
}

\author{
Stephen J. Smartt \\ Institute of Astronomy, University of Cambridge, \\ Madingley Road, Cambridge CB3 OHA, UK
}

\begin{abstract}
A novel project to directly detect the progenitor stars of nearby
\end{abstract} core-collapse supernovae in pre-discovery archive images is briefly described.

\section{Summary of project}

The masses and the evolutionary states of the progenitors of core-collapse $\mathrm{SNe}$ are not well constrained by direct observations. Stellar evolution theory generally predicts, that massive stars with initial masses less than about $30 \mathrm{M}_{\odot}$ should undergo core-collapse, when they are cool M-type supergiants. However, the only two detections of a SN progenitor are SN 1987A and SN 1993J, and neither of these was an M-type supergiant. I have started a novel project, which may allow the direct identification of core-collapse SNe progenitors on pre-explosion images of resolved, nearby galaxies. This project is now possible, with the excellent image archives maintained by several facilities and will be enhanced by new initiatives to create Virtual Observatories, the earliest of which (ASTROVIRTEL ${ }^{1}$ ) is already producing results. Pre-discovery images from the HST of two Type II-P SNe (SN 1999gi and SN 2001du) within $20 \mathrm{Mpc}$ of the Milky Way, show no sign of a progenitor star. In addition, two of the closest $\mathrm{SNe}$ in recent years have high quality pre-explosion ground-based images (SN 1999em and SN 2002ap) and, again, there is no detection of a stellar progenitor. However, stringent upper mass and luminosity limits can be determined for the exploding stars.

\section{Examples of events: SNe 1999gi, 1999em, 2001du and 2002ap}

The bright SN 1999gi was discovered in NGC 3184 ( 8 Mpc). It was a Type II-P, with a peak $\mathrm{M}_{V} \simeq-16$, a $\sim 100 \mathrm{~d}$ plateau, and was a faint $\mathrm{X}$-ray and radio source (Smartt et al. 2001). By chance, there are archive images of this galaxy taken several years before explosion by HST. Images taken after explosion allow the SN position to be precisely determined on the pre-explosion frames. However there is no detection of a progenitor star at the $\mathrm{SN}$ position. By measuring the sensitivity limits of the images, the bolometric luminosity limits of the progenitors (as a function of stellar effective temperature) can been determined. Comparing these with stellar evolutionary tracks, allows one to estimate the initial mass of the

\footnotetext{
${ }^{1}$ http://www.stecf.org/astrovirtel
} 
Table 1. Summary of what we know about SN progenitors, taken from Smartt et al. (2002a, 2002b, 2002c in preparation). The metallicity, expressed as a fraction of solar $\left(\mathrm{Z}_{\odot}\right)$, is generally taken from $\mathrm{H}$ II regions in the vicinity of the $\mathrm{SNe}$ or estimated from the abundance gradients in the spirals.

\begin{tabular}{|c|c|c|c|c|c|c|}
\hline \multirow[t]{2}{*}{$\mathrm{SN}$} & \multirow[t]{2}{*}{ galaxy } & \multirow{2}{*}{$\begin{array}{c}\text { distance } \\
(\mathrm{Mpc})\end{array}$} & \multirow[t]{2}{*}{ type } & \multirow{2}{*}{$\begin{array}{c}Z \\
\left(\mathrm{Z}_{\odot}\right)\end{array}$} & \multicolumn{2}{|c|}{ progenitor } \\
\hline & & & & & type & mass \\
\hline SN 1987A & LMC & 0.05 & II-pec & 0.5 & B3Ia & $20 \mathrm{M}_{\odot}$ \\
\hline SN 1993J & M81 & 3.6 & IIb & 2 & $\mathrm{K} 0 \mathrm{Ia}$ & $17 \mathrm{M}_{\odot}$ \\
\hline SN 1980K & NGC6946 & 5.1 & II-L & 0.5 & $?$ & $<20 \mathrm{M}_{\odot}$ \\
\hline SN $1999 \mathrm{em}$ & NGC1637 & 7.5 & II-P & $1-2$ & M-type? & $<12 \mathrm{M}_{\odot}$ \\
\hline SN 1999gi & NGC3184 & 7.9 & II-P & $\sim 2$ & M-type? & $<9 \mathrm{M}_{\odot}$ \\
\hline SN 2001du & NGC1365 & 17.2 & II-P & $\sim$ & M-type? & $<12 \mathrm{M}_{\odot}$ \\
\hline SN 2002ap & NGC628 & 7.3 & Ic & 0.5 & WR? & $<30 \mathrm{M}_{\odot}$ \\
\hline
\end{tabular}

progenitor. In Smartt et al. (2001) we have derived an upper mass limit of $9_{-2}^{+3} \mathrm{M}_{\odot}$. We have derived similar mass limits for SN 1999em and SN 2001du (see Table 1), with uncertainties of roughly $\pm 3 \mathrm{M}_{\odot}$. These three $\mathrm{SNe}$ are very similar in their observed characteristics and have rather similar mass limits. The low values of the X-ray and radio fluxes suggest that the progenitor stars of SN 1999gi and SN $1999 \mathrm{em}$ had relatively low mass-loss rates, which is consistent with the fairly low masses we derive. The stars probably had initial masses of 9-12 $\mathrm{M}_{\odot}$ and exploded as moderate mass red-supergiants.

We have studied pre-explosion images of the Type Ic hypernova SN 2002ap (Mazzali et al. 2002), and again found no detection of a progenitor. The deepest of these images is the $B$-band exposure, which has a sensitivity limit of $M_{B}<-6.3$ (Smartt et al. 2002b). We concluded that the progenitor was either a Wolf-Rayet star or a $\sim 20-25 \mathrm{M}_{\odot}$ star in an interacting binary system which has had its outer $\mathrm{H}$-rich envelope removed due to mass transfer.

These examples show how having high-quality archive images of SNe sites taken prior to explosion, allow stringent limits to be set on the nature of the progenitor stars. Smartt et al. (2002a) have estimated that $\sim 2.4 \pm 2 \mathrm{SNe} \mathrm{yr}^{-1}$ will have pre-explosion information and hence a project lasting 3-5 years should significantly improve our knowledge. I currently have an HST T-o-O programme (Cycles 10 and 11) to re-observe interesting events, in order to do very accurate astrometric positioning of the $\mathrm{SN}$ on high resolution predisovery images.

\section{References}

Mazzali, P., Deng, J., Maeda, K., et al. 2002, ApJ (Letters) 572, L61

Smartt, S.J., Gilmore, G.F., Trentham, N., Tout, C.A., Frayn, C.M. 2001, ApJ (Letters) $556, \mathrm{~L} 29$

Smartt, S.J., Gilmore, G.F., Tout, C.A., Hodgkin, S.T. 2002a, ApJ 565, 1089

Smartt, S.J., Vreeswijk, P.M., Ramirez-Ruiz, E., Gilmore, G.F., Meikle, W.P.S., Ferguson, A.M.N., Knapen, J. 2002b, ApJ (Letters) 572, L147 